\title{
Unsteady Solutions in a Third-Grade Fluid Filling the Porous Space
}

\author{
T. Hayat, ${ }^{1}$ H. Mambili-Mamboundou, ${ }^{2}$ and F. M. Mahomed ${ }^{2}$ \\ ${ }^{1}$ Department of Mathematics, Quaid-i-Azam University 45320, Islamabad 44000, Pakistan \\ ${ }^{2}$ Centre for Differential Equations, Continuum Mechanics, and Applications, School of Computational \\ and Applied Mathematics, University of the Witwatersrand, Wits 2050, South Africa
}

Correspondence should be addressed to F. M. Mahomed, fazal.mahomed@wits.ac.za

Received 28 August 2007; Accepted 15 May 2008

Recommended by Horst Ecker

An analysis is made of the unsteady flow of a third-grade fluid in a porous medium. A modified Darcy's law is considered in the flow modelling. Reduction and solutions are obtained by employing similarity and numerical methods. The effects of pertinent parameters on the flow velocity are studied through graphs.

Copyright $@ 2008$ T. Hayat et al. This is an open access article distributed under the Creative Commons Attribution License, which permits unrestricted use, distribution, and reproduction in any medium, provided the original work is properly cited.

\section{Introduction}

Recently, it has been recognised in industrial and technological applications that nonNewtonian fluids are more appropriate than viscous fluids. However, there is no model which can alone predict the behaviour of all non-Newtonian fluids. The governing equations of nonNewtonian fluids are of higher order than the Navier-Stokes equations. Therefore, the adhering boundary conditions are not sufficient, and one needs additional boundary conditions for a unique solution. Excellent critical reviews in this direction have been given by Rajagopal [1,2], Rajagopal et al. [3], and Rajagopal and Kaloni [4]. Amongst the several non-Newtonian fluid models, much attention has been paid to the simplest subclass of viscoelastic fluids known as the second grade. However, this model is not capable of describing the shear thinning and thickening phenomena for steady flow over a rigid boundary. The third-grade fluid model represents a further, although inconclusive, attempt towards a more comprehensive description of the behaviour of viscoelastic fluids. Also, the flows of such fluids in porous medium are quite prevalent in many engineering fields such as enhanced oil recovery, paper and textile coating, and composite manufacturing processes. Some important studies dealing with the flows of non-Newtonian fluids are made by Rajagopal and Na [5, 6], Rajagopal and 
Gupta [7], Hayat et al. [8-11], Hayat and Ali [12], Ariel et al. [13], Hayat and Kara [14], AbdelMalek et al. [15], Wafo Soh [16] and Chen et al. [17], and Fetecau and Fetecau [18-20].

Recently, Tan and Masuoka [21] analysed the Stokes' first problem for a second-grade fluid in a porous medium. In another paper, Tan and Masuoka [22] studied the Stokes' first problem for an Oldroyd-B fluid in a porous medium. In these investigations, the authors have used the modified Darcy's law.

The main goal of this paper is to determine analytical solutions for an unsteady flow of a third-grade fluid over a moved plate. The relevant problem is formulated using modified Darcy's law of a third-grade fluid. Two types of analytical solutions are presented and discussed. A numerical solution is also presented.

\section{Problem formulation}

Let us introduce a Cartesian coordinate system OXYZ with $y$-axis in the upward direction. The third-grade fluid fills the porous space $y>0$ and is in contact with an infinite moved plate at $y=0$. For unidirectional flow, the velocity field is

$$
\mathbf{V}=(u(y, t), 0,0),
$$

where the above definition of velocity automatically satisfies the incompressibility condition. The equation of motion in a porous medium without body forces is

$$
\rho \frac{d \mathbf{V}}{d t}=\operatorname{div} \mathbf{T}+\mathbf{r}
$$

where $\rho$ is the fluid density, $d / d t$ is the material time differentiation, $T$ is the Cauchy stress tensor, and $\mathbf{r}$ is the Darcy's resistance in a porous space. The Cauchy stress tensor of an incompressible third-grade fluid has the form [23]

$$
\mathbf{T}=-p \mathbf{I}+\mu \mathbf{A}_{1}+\alpha_{1} \mathbf{A}_{2}+\alpha_{2} \mathbf{A}_{1}^{2}+\beta_{1} \mathbf{A}_{3}+\beta_{2}\left(\mathbf{A}_{1} \mathbf{A}_{2}+\mathbf{A}_{2} \mathbf{A}_{1}\right)+\beta_{3}\left(\operatorname{tr} \mathbf{A}_{1}^{2}\right) \mathbf{A}_{1}
$$

in which $p$ is the pressure, $\mathbf{I}$ is the identity tensor, $\alpha_{i}(i=1,2)$ and $\beta_{i}(i=1-3)$ are the material constants, and $\mathbf{A}_{i}(i=1-3)$ are the first Rivlin-Ericksen tensors [24] which may be defined through the following equations:

$$
\begin{gathered}
\mathbf{A}_{1}=(\operatorname{grad} \mathbf{V})+(\operatorname{grad} \mathbf{V})^{T}, \\
\mathbf{A}_{n}=\frac{d \mathbf{A}_{n-1}}{d t}+\mathbf{A}_{n-1}(\operatorname{grad} \mathbf{V})+(\operatorname{grad} \mathbf{V})^{T} \mathbf{A}_{n-1} ; \quad n>1 .
\end{gathered}
$$

In studying fluid dynamics, it is assumed that the flow meets the Clausius-Duhem inequality and that the specific Helmholtz free energy of the fluid is a minimum at equilibrium when [25]

$$
\begin{gathered}
\mu \geq 0, \quad \alpha_{1} \geq 0, \quad \beta_{1}=\beta_{2}=0, \quad \beta_{3} \geq 0, \\
\left|\alpha_{1}+\alpha_{2}\right| \leq \sqrt{24 \mu \beta_{3} .}
\end{gathered}
$$

On the basis of constitutive equation in an Oldroyd-B fluid, the following expression in a porous medium has been proposed [26]:

$$
\left(1+\lambda \frac{\partial}{\partial t}\right) \nabla p=-\frac{\mu \phi}{k}\left(1+\lambda_{r} \frac{\partial}{\partial t}\right) \mathbf{V}
$$


where $\lambda$ and $\lambda_{r}$ are the relaxation and retardation times, and $\phi$ and $k$ are the porosity and permeability of the porous medium, respectively. It should be pointed out that for $\lambda_{r}=0$, (2.6) reduces to the expression which holds for a Maxwell fluid [26] and when $\lambda=0$, it reduces to that of second-grade fluid [22].

Keeping the analogy of (2.6) with the constitutive equation of an extra stress tensor in an Oldroyd-B fluid, the following expression in the present problem has been suggested:

$$
\frac{\partial p}{\partial x}=-\frac{\phi}{k}\left[\mu+\alpha_{1} \frac{\partial}{\partial t}+2 \beta_{3}\left(\frac{\partial u}{\partial y}\right)^{2}\right] u \text {. }
$$

Since the pressure gradient in (2.7) can also be interpreted as a measure of the flow resistance in the bulk of the porous medium, and $r_{x}$ is the measure of the flow resistance offered by the solid matrix in $x$-direction, then

$$
r_{x}=-\frac{\phi}{k}\left[\mu+\alpha_{1} \frac{\partial}{\partial t}+2 \beta_{3}\left(\frac{\partial u}{\partial y}\right)^{2}\right] u
$$

From (2.1) to (2.5) and (2.8), we have

$$
\rho \frac{\partial u}{\partial t}=\mu \frac{\partial^{2} u}{\partial y^{2}}+\alpha_{1} \frac{\partial^{3} u}{\partial y^{2} \partial t}+6 \beta_{3}\left(\frac{\partial u}{\partial y}\right)^{2} \frac{\partial^{2} u}{\partial y^{2}}-\left[\mu+\alpha_{1} \frac{\partial}{\partial t}+2 \beta_{3}\left(\frac{\partial u}{\partial y}\right)^{2}\right] \frac{\phi u}{k} .
$$

The relevant boundary and initial conditions are

$$
\begin{gathered}
u(0, t)=u_{0} V(t), \quad t>0, \\
u(\infty, t)=0, \quad t>0, \\
u(y, 0)=g(y), \quad y>0,
\end{gathered}
$$

in which $u_{0}$ is the reference velocity.

\section{Solutions of the problem}

We rewrite (2.9) as

$$
\frac{\partial u}{\partial t}=\mu_{*} \frac{\partial^{2} u}{\partial y^{2}}+\alpha \frac{\partial^{3} u}{\partial y^{2} \partial t}+\gamma_{1}\left(\frac{\partial u}{\partial y}\right)^{2} \frac{\partial^{2} u}{\partial y^{2}}-\gamma_{2} u\left(\frac{\partial u}{\partial y}\right)^{2}-\phi_{1} u
$$

where

$$
\begin{gathered}
\mu_{*}=\frac{\mu}{\rho+\alpha_{1}(\phi / k)}, \quad \alpha=\frac{\alpha_{1}}{\rho+\alpha_{1}(\phi / k)}, \quad \gamma_{1}=\frac{6 \beta_{3}}{\rho+\alpha_{1}(\phi / k)}, \\
\gamma_{2}=\frac{2 \beta_{3}(\phi / k)}{\rho+\alpha_{1}(\phi / k)}, \quad \phi_{1}=\frac{\mu(\phi / k)}{\rho+\alpha_{1}(\phi / k)} .
\end{gathered}
$$




\subsection{Lie symmetry analysis}

The Lie symmetry analysis reveals that (3.1) admits two sets of symmetry generators depending on the value of $\phi_{1}$. The appendix provides details of the symmetry analysis of (3.1). Case $1\left(\phi_{1} \neq \mu_{*} / \alpha\right)$. We obtain a two-dimensional Lie algebra generated by

$$
X_{1}=\frac{\partial}{\partial y}, \quad X_{2}=\frac{\partial}{\partial t} .
$$

Case $2\left(\phi_{1}=\mu_{*} / \alpha\right)$. We find a three-dimensional Lie algebra generated by

$$
X_{1}=\frac{\partial}{\partial y}, \quad X_{2}=\frac{\partial}{\partial t}, \quad X_{3}=e^{\left(2 \mu_{*} / \alpha\right) t} \frac{\partial}{\partial t}-\frac{\mu_{*}}{\alpha} e^{\left(2 \mu_{*} / \alpha\right) t} u \frac{\partial}{\partial u} .
$$

\subsection{Travelling wave solutions}

We now look for invariant solutions under the operator $X_{1}-c X_{2}$, which represents wave-fronttype travelling wave solutions with constant wave speed $c$. The invariant is given by

$$
u(y, t)=U\left(x_{1}\right), \quad \text { where } x_{1}=y+c t .
$$

Substituting (3.5) into (3.1) yields a third-order ordinary differential equation for $U\left(x_{1}\right)$,

$$
\phi_{1} U\left(x_{1}\right)=-c \frac{d U}{d x_{1}}-\gamma_{2} U\left(x_{1}\right)\left(\frac{d U}{d x_{1}}\right)^{2}+\mu \frac{d^{2} U}{d x_{1}^{2}}+r_{1}\left(\frac{d U}{d x_{1}}\right)^{2} \frac{d^{2} U}{d x_{1}^{2}}+c \alpha \frac{d^{3} U}{d x_{1}^{3}} .
$$

It can be seen that this equation admits the solution

$$
U\left(x_{1}\right)=u_{0} \exp \left(\frac{\sqrt{\gamma_{2}} x_{1}}{-\sqrt{\gamma_{1}}}\right)
$$

provided that

$$
\frac{\gamma_{2}}{\gamma_{1}}\left(\mu-c \alpha \sqrt{\frac{\gamma_{2}}{\gamma_{1}}}\right)+\left(c \alpha \sqrt{\frac{\gamma_{2}}{\gamma_{1}}}-\phi_{1}\right)=0,
$$

and hence (3.1) subject to (2.10)-(2.12) admits the solution

$$
u(y, t)=u_{0} \exp \left(\frac{\sqrt{\gamma_{2}}(y+c t)}{-\sqrt{\gamma_{1}}}\right) .
$$

This solution is plotted in Figures 1 and 4 for various values of the emerging parameters.

On the other hand, we find group-invariant solutions corresponding to operators which give meaningful physical solutions of the initial and boundary value problems (2.9) to (2.12). This means $X_{2}$ and $X_{3}$.

\subsection{Group-invariant solutions corresponding to $X_{2}$}

The invariant solution admitted by $X_{2}$ is the steady-state solution

$$
u(y, t)=F(y) .
$$

The substitution of (3.10) into (3.1) yields the second-order ordinary differential equation for $F(y)$ :

$$
\gamma_{1}\left(F^{\prime}(y)\right)^{2} F^{\prime \prime}(y)+\mu_{*} F^{\prime \prime}(y)-\gamma_{2} F(y)\left(F^{\prime}(y)\right)^{2}-\phi_{1} F(y)=0
$$


Variation of the flow with time, with $\gamma_{1}=1.5$,
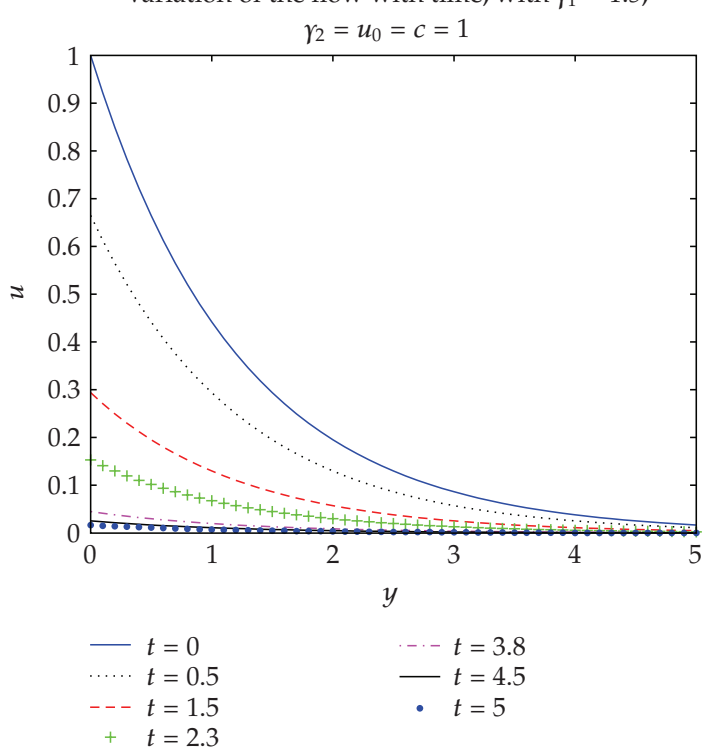

Figure 1: Travelling wave solutions varying $t$.

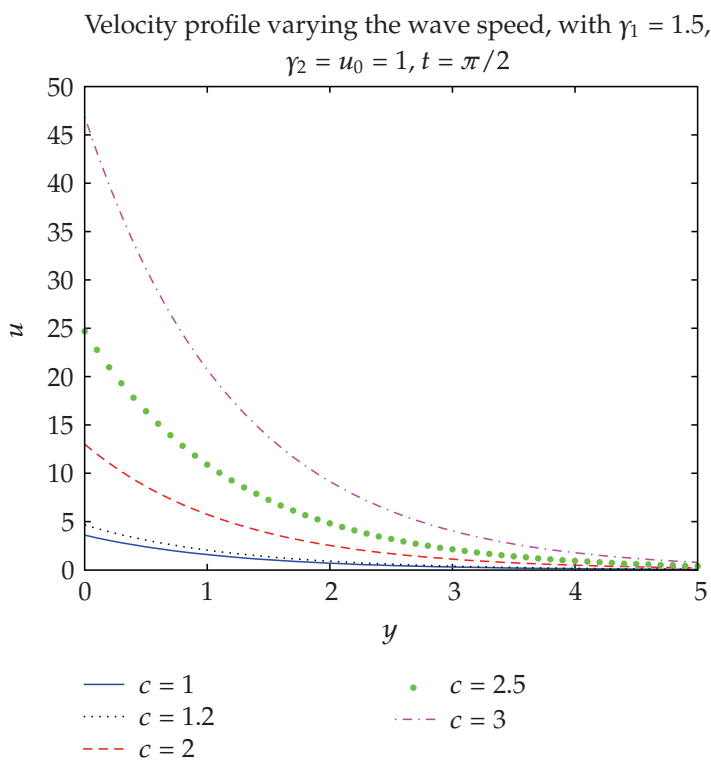

Figure 2: Travelling wave solutions varying $c$.

subject to boundary conditions

$$
\begin{gathered}
F(0)=v_{0}, \\
F(l)=0, \quad l>0,
\end{gathered}
$$




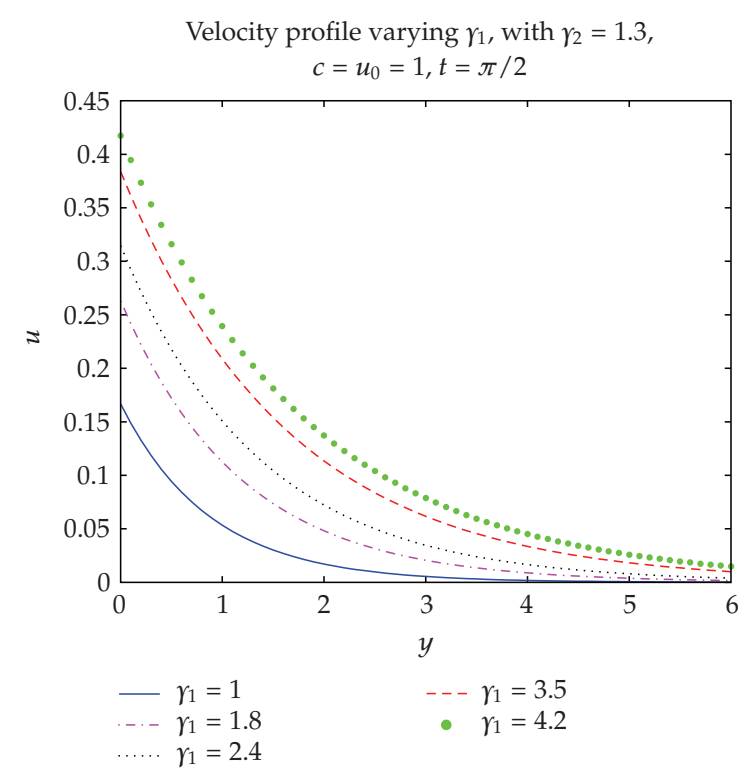

Figure 3: Travelling wave solutions varying $\gamma_{1}$.

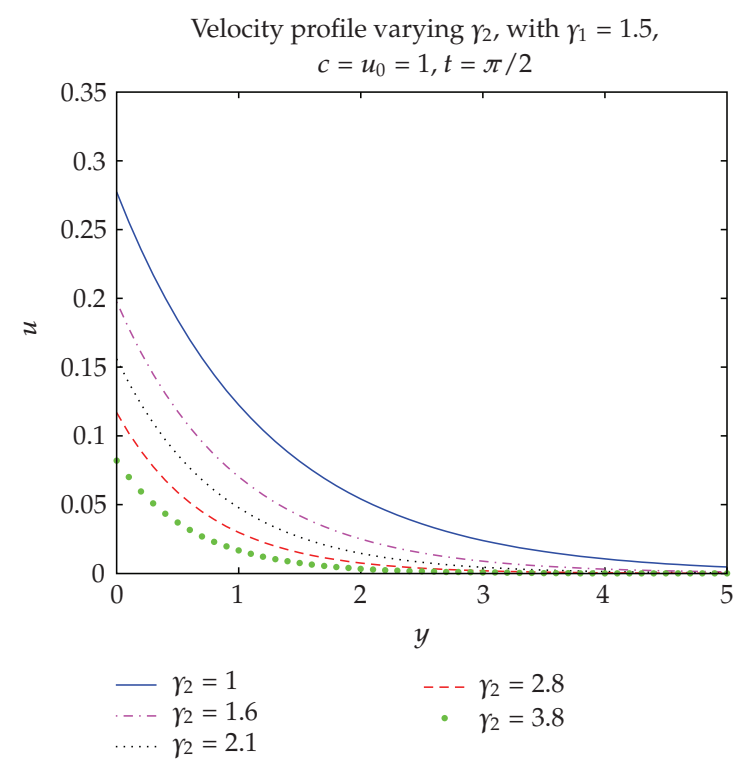

Figure 4: Travelling wave solutions varying $\gamma_{2}$.

where $l$ is sufficiently large, and $u_{0} V=v_{0}$ is a constant ( $V$ is taken to be a constant). Let

$$
K(F)=\frac{d F}{d y}
$$




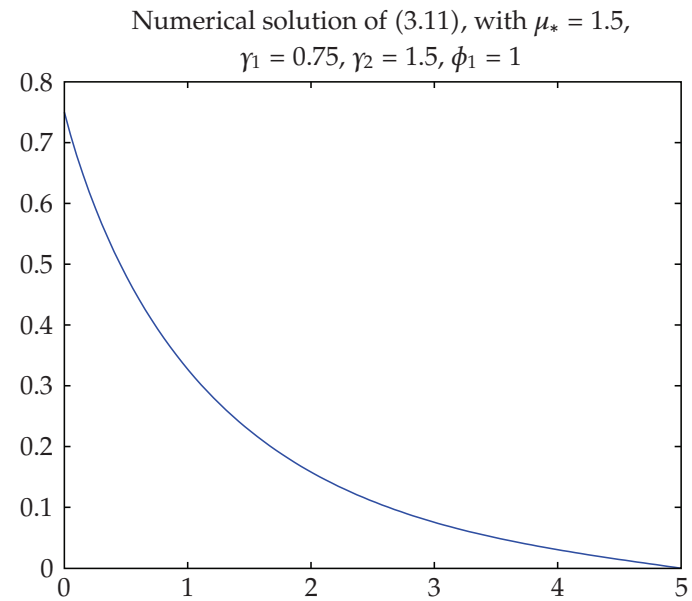

Figure 5: Numerical solution of (3.11) or (3.17) subject to the boundary conditions (3.12) and (3.13).

then (3.11) transforms to

$$
\gamma_{1} K(F)^{3} K^{\prime}(F)+\mu_{*} K(F) K^{\prime}(F)-\gamma_{2} F K(F)^{2}-\phi_{1} F=0 .
$$

The integration of (3.15) gives

$$
\frac{\gamma_{1}}{\gamma_{2}} K(F)+\frac{\mu_{*} \gamma_{2}-\gamma_{1} \phi_{1}}{\gamma_{2} \sqrt{\gamma_{2} \phi_{1}}}, \quad \operatorname{Arctan}\left[\sqrt{\frac{\gamma_{2}}{\gamma_{1}}} K(F)\right]=\frac{1}{2} y^{2}+C,
$$

where $C$ is a constant. Equation (3.16) is equivalent to the following first-order ODE in $F$ :

$$
\frac{\gamma_{1}}{\gamma_{2}} F^{\prime}(y)+\frac{\mu_{*} \gamma_{2}-\gamma_{1} \phi_{1}}{\gamma_{2} \sqrt{\gamma_{2} \phi_{1}}}, \quad \operatorname{Arctan}\left[\sqrt{\frac{\gamma_{2}}{\gamma_{1}}} F^{\prime}(x)\right]=\frac{1}{2} y^{2}+C .
$$

One can solve this numerically subject to the boundary conditions (3.12) and (3.13). This solution is plotted in Figure 5.

\subsection{Group-invariant solutions corresponding to $X_{3}$}

The invariant solution admitted by $X_{3}$ is

$$
u=u_{0} \exp \left(\frac{-\mu_{*}}{\alpha} t\right) B(y)
$$

where $B(y)$ as yet is an undetermined function of $y$. Substituting (3.18) into (3.1) yields the linear second-order ordinary differential equation

$$
B^{\prime \prime}-\frac{\gamma_{2}}{r_{1}} B=0
$$




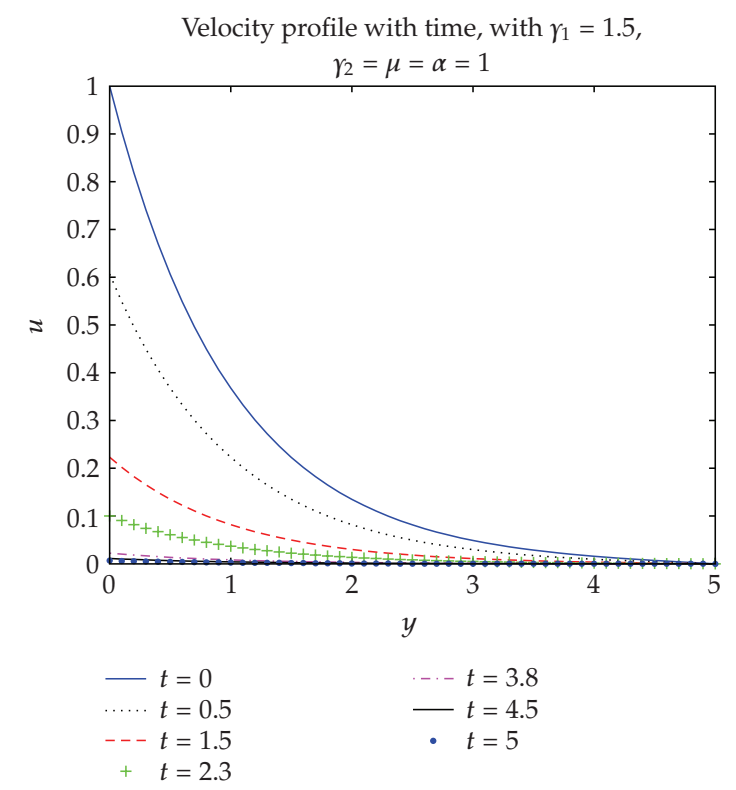

Figure 6: Analytical solutions for $\mu_{*}=1, u_{0}=1$.

From (2.10) to (2.11), the appropriate boundary conditions for (3.19) are

$$
B(0)=1, \quad B(l)=0, \quad l \rightarrow \infty,
$$

where

$$
V(t)=u_{0} \exp \left(\frac{-\mu_{*}}{\alpha} t\right)
$$

We solve (3.19) subject to the boundary conditions (3.20) for positive $\gamma_{2} / \gamma_{1}$ and obtain

$$
B=\exp \left(-\sqrt{\frac{\gamma_{2}}{\gamma_{1}}} y\right)
$$

The solutions (3.18) are plotted for positive $\gamma_{2} / \gamma_{1}$ in Figure 6 . This solution is similar to (3.9) except that we do not have a condition like (3.8) here.

\subsection{Numerical solution}

We present the numerical solution of (3.1) subject to the initial and boundary conditions:

$$
\begin{gathered}
u(0, t)=u_{0} V(t), \quad u(\infty, t)=0, \quad t>0, \\
u(y, 0)=g(y), \quad y>0,
\end{gathered}
$$

where $g(y)$ is an arbitrary function of $y$.

This solution is plotted using Mathematica's solver NDSolve. 


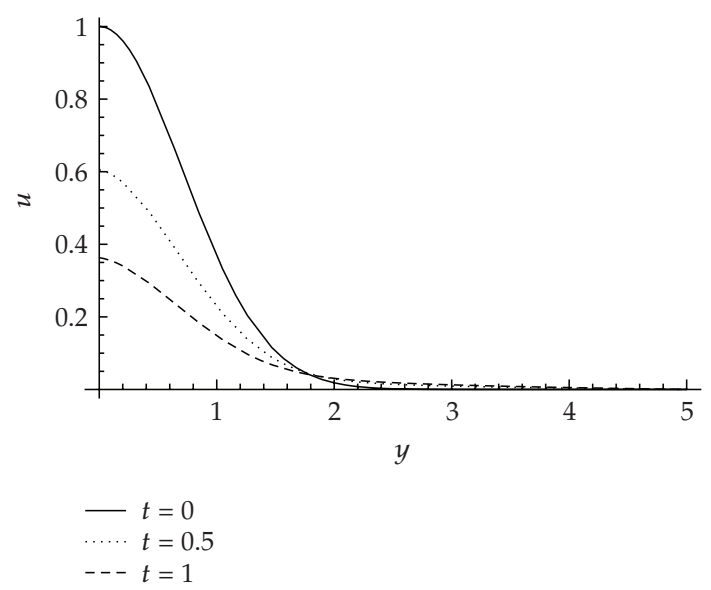

Figure 7: Numerical solution of (3.1), with $V(t)=e^{-t}, g(y)=e^{-y^{2}}, \mu_{*}=2.5, u_{0}=1, \alpha=2, \gamma_{1}=1.5, \gamma_{2}=2.6$, $\phi_{1}=0.8$.

\section{Results and discussion}

In order to see the variation of various physical parameters on the velocity, Figures 1-7 have been plotted.

The effect of unsteadiness on the velocity profile is shown in Figure 1 . This figure depicts that velocity decreases for large values of time. Clearly, the variation of velocity is observed for $0 \leq t<3.8$. For $t \geq 3.8$, the velocity profile remains the same. In other words, one can say that steady-state behaviour is achieved for $t \geq 3.8$.

The influence of the wave speed $c$ on the velocity profile has been presented in Figure 2 . It is revealed that velocity decreases by increasing $c$. Moreover, the effects of the fluid parameters $\gamma_{1}$ and $\gamma_{2}$ are given in Figures 3 and 4, respectively. These figures depict that $\gamma_{1}$ and $\gamma_{2}$ have opposite roles on the velocity. These figures show that velocity increases for large values of $\gamma_{1}$ whereas it decreases for increasing $\gamma_{2}$. In Figure 5, the steady-state solution is plotted, and the velocity profile is the same as observed in the case of travelling wave solution.

Further, the analytical solutions (3.19) for $\mu_{*} / \alpha>0$ is plotted in Figure 6. Here as indicated in Figure 6, the velocity profile decreases for large values of $t$. Ultimately when $t \geq 3.8$, there is almost no variation in velocity.

Finally in Figure 7, we have plotted numerically the velocity profile for small variations of time, and it is observed that the velocity decreases as time increases, which is the the same observation made previously for the analytical solutions.

\section{Appendix}

The operator

$$
X=\tau(t, y, u) \frac{\partial}{\partial t}+\xi(t, y, u) \frac{\partial}{\partial y}+\eta(t, y, u) \frac{\partial}{\partial u}
$$

is a generator of Lie point symmetry of (3.1) if

$$
\left.X^{[3]}\left(u_{t}-\mu_{*} u_{y y}-\alpha u_{t y y}-\gamma_{1}\left(u_{y}\right)^{2} u_{y y}+\gamma_{2} u\left(u_{y}\right)^{2}+\phi_{1} u\right)\right|_{(14)}=0,
$$


where

$$
X^{[3]}=X+\eta^{t} \frac{\partial}{\partial u_{t}}+\eta^{y} \frac{\partial}{\partial u_{y}}+\eta^{y y} \frac{\partial}{\partial u_{y y}}+\eta^{t y y} \frac{\partial}{\partial u_{t y y}}
$$

in which

$$
\begin{gathered}
\eta^{t}=D_{t} \eta-u_{t} D_{t} \tau-u_{y} D_{t} \xi, \\
\eta^{y}=D_{y} \eta-u_{t} D_{y} \tau-u_{y} D_{y} \xi, \\
\eta^{y y}=D_{y} \eta^{y}-u_{t y} D_{y} \tau-u_{y y} D_{y} \xi, \\
\eta^{t y y}=D_{t} \eta^{y y}-u_{t y y} D_{t} \tau-u_{y y y} D_{t} \xi
\end{gathered}
$$

and the total derivative operators are

$$
\begin{aligned}
& D_{t}=\frac{\partial}{\partial t}+u_{t} \frac{\partial}{\partial u}+u_{t t} \frac{\partial}{\partial u_{t}}+\cdots \\
& D_{y}=\frac{\partial}{\partial y}+u_{y} \frac{\partial}{\partial u}+u_{y y} \frac{\partial}{\partial u_{y}}+\cdots
\end{aligned}
$$

Substituting the expansion of (A.4) into the symmetry condition (A.2) and separating them by powers of the derivatives of $u$, since $\tau, \xi$, and $\eta$ are independent of the derivatives of $u$, lead to the overdetermined system of linear partial differential equations (note that $\gamma_{1}$ and $\gamma_{2}$ are not zero):

$$
\begin{gathered}
\tau_{y}=\tau_{u}=0, \\
\xi_{t}=\xi_{y}=\xi_{u}=0, \\
\eta_{x}=\eta_{u u}=0, \\
\tau_{t} \mu_{*}+\alpha \eta_{t u}=0, \\
u \eta_{u}+\tau_{t} u+\eta=0, \\
\eta_{t}+\phi_{1} \eta+\phi_{1} u\left(\tau_{t}-\eta_{u}\right)=0 .
\end{gathered}
$$

The solution of this linear system (A.6) gives rise to two cases $\phi_{1} \neq \mu_{*} / \alpha$ and $\phi_{1}=\mu_{*} / \alpha$. In the former, we obtain

$$
\xi=a_{1}, \quad \tau=a_{2}, \quad \eta=0,
$$

and for the second case

$$
\xi=a_{1}, \quad \tau=-\frac{a_{2}}{\phi_{1}} \exp \left(2 \phi_{1} t\right)+a_{3}, \quad \eta=a_{2} u \exp \left(2 \phi_{1} t\right) .
$$

In both (A.7) and (A.8), the $a_{i}$ are constants. Setting one of the constants $a_{i}$ equal to one and the rest of the constants to zero results in the generators given in Section 3.1. 


\section{References}

[1] K. R. Rajagopal, "Boundedness and uniqueness of fluids of the differential type," Acta Ciencia Indica, vol. 8, no. $1-4$, pp. 28-38, 1982.

[2] K. R. Rajagopal, "On boundary conditions for fluids of the differential type," in Navier-Stokes Equations and Related Nonlinear Problems, A. Sequira, Ed., pp. 273-278, Plenum Press, New York, NY, USA, 1995

[3] K. R. Rajagopal, A. Z. Szeri, and W. Troy, "An existence theorem for the flow of a non-Newtonian fluid past an infinite porous plate," International Journal of Non-Linear Mechanics, vol. 21, no. 4, pp. 279-289, 1986.

[4] K. R. Rajagopal and P. N. Kaloni, "Some remarks on boundary conditions for flows of fluids of the differential type," in Continuum Mechanics and Its Applications, G. A. C. Graham and S. K. Malik, Eds., pp. 935-942, Hemisphere, New York, NY, USA, 1989.

[5] K. R. Rajagopal and T. Y. Na, “On Stokes' problem for a non-Newtonian fluid," Acta Mechanica, vol. 48, no. 3-4, pp. 233-239, 1983.

[6] K. R. Rajagopal and T. Y. Na, "Natural convection flow of a non-Newtonian fluid between two vertical flat plates," Acta Mechanica, vol. 54, no. 3-4, pp. 239-246, 1985.

[7] K. R. Rajagopal and A. S. Gupta, "An exact solution for the flow of a non-Newtonian fluid past an infinite porous plate," Meccanica, vol. 19, no. 2, pp. 158-160, 1984.

[8] T. Hayat, M. Khan, and M. Ayub, "Some analytical solutions for second grade fluid flows for cylindrical geometries," Mathematical and Computer Modelling, vol. 43, no. 1-2, pp. 16-29, 2006.

[9] T. Hayat, Z. Abbas, and S. Asghar, "Heat transfer analysis on rotating flow of a second-grade fluid past a porous plate with variable suction," Mathematical Problems in Engineering, vol. 2005, no. 5, pp. 555-582, 2005.

[10] T. Hayat, S. Nadeem, S. Asghar, and A. M. Siddiqui, "Effects of Hall current on unsteady flow of a second grade fluid in a rotating system," Chemical Engineering Communications, vol. 192, no. 10, pp. 1272-1284, 2005.

[11] T. Hayat, R. Ellahi, P. D. Ariel, and S. Asghar, "Homotopy solution for the channel flow of a third grade fluid," Nonlinear Dynamics, vol. 45, no. 1-2, pp. 55-64, 2006.

[12] T. Hayat and N. Ali, "Peristaltically induced motion of a MHD third grade fluid in a deformable tube," Physica A, vol. 370, no. 2, pp. 225-239, 2006.

[13] P. D. Ariel, T. Hayat, and S. Asghar, "The flow of an elastico-viscous fluid past a stretching sheet with partial slip," Acta Mechanica, vol. 187, no. 1-4, pp. 29-35, 2006.

[14] T. Hayat and A. H. Kara, "Couette flow of a third-grade fluid with variable magnetic field," Mathematical and Computer Modelling, vol. 43, no. 1-2, pp. 132-137, 2006.

[15] M. B. Abd-el-Malek, N. A. Badran, and H. S. Hassan, "Solution of the Rayleigh problem for a power law non-Newtonian conducting fluid via group method," International Journal of Engineering Science, vol. 40, no. 14, pp. 1599-1609, 2002.

[16] C. Wafo Soh, "Invariant solutions of the unidirectional flow of an electrically charged power-law non-Newtonian fluid over a flat plate in presence of a transverse magnetic field," Communications in Nonlinear Science and Numerical Simulation, vol. 10, no. 5, pp. 537-548, 2005.

[17] C.-I. Chen, T. Hayat, and J.-L. Chen, "Exact solutions for the unsteady flow of a Burger's fluid in a duct induced by time-dependent prescribed volume flow rate," Heat and Mass Transfer, vol. 43, no. 1, pp. 85-90, 2006.

[18] C. Fetecau and C. Fetecau, "Starting solutions for the motion of a second grade fluid due to longitudinal and torsional oscillations of a circular cylinder," International Journal of Engineering Science, vol. 44, no. 11-12, pp. 788-796, 2006.

[19] C. Fetecau and C. Fetecau, "Decay of a potential vortex in an Oldroyd-B fluid," International Journal of Engineering Science, vol. 43, no. 3-4, pp. 340-351, 2005.

[20] C. Fetecau and C. Fetecau, "Starting solutions for some unsteady unidirectional flows of a second grade fluid," International Journal of Engineering Science, vol. 43, no. 10, pp. 781-789, 2005.

[21] W. Tan and T. Masuoka, "Stokes' first problem for a second grade fluid in a porous half-space with heated boundary," International Journal of Non-Linear Mechanics, vol. 40, no. 4, pp. 515-522, 2005.

[22] W. Tan and T. Masuoka, "Stokes' first problem for an Oldroyd-B fluid in a porous half space," Physics of Fluids, vol. 17, no. 2, Article ID 023101, 7 pages, 2005.

[23] C. Truesdell and W. Noll, "The non-linear field theories of mechanics," in Handbuch der Physik, Band III/3, pp. 1-602, Springer, Berlin, Germany, 1965. 
[24] R. S. Rivlin and J. L. Ericksen, "Stress-deformation relations for isotropic materials," Journal of Rational Mechanics and Analysis, vol. 4, pp. 323-425, 1955.

[25] R. L. Fosdick and K. R. Rajagopal, "Thermodynamics and stability of fluids of third grade," Proceedings of the Royal Society of London, vol. 369, no. 1738, pp. 351-377, 1980.

[26] M. G. Alishayev, "Proceedings of Moscow Pedagogy Institute," Hydrodynamics, vol. 3, pp. 166-174, 1974 (Russian). 


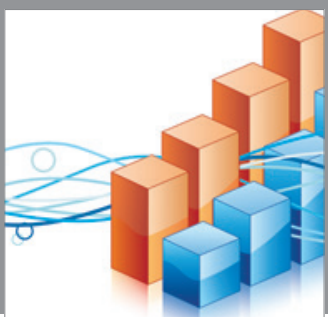

Advances in

Operations Research

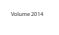

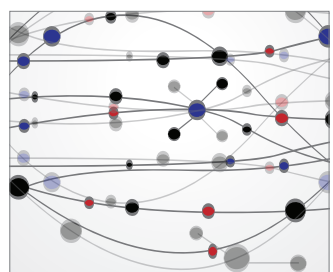

\section{The Scientific} World Journal
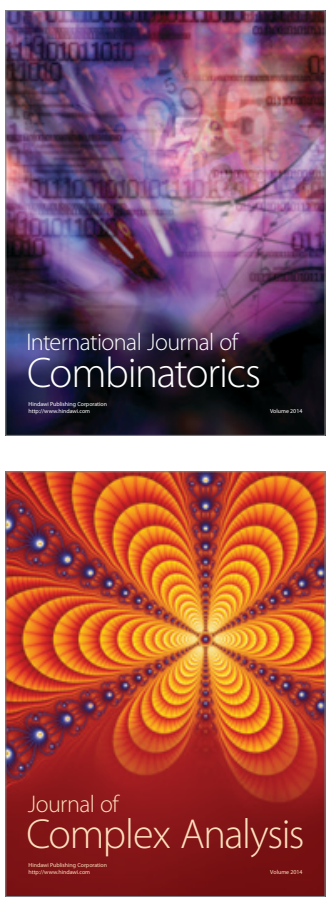

International Journal of

Mathematics and

Mathematical

Sciences
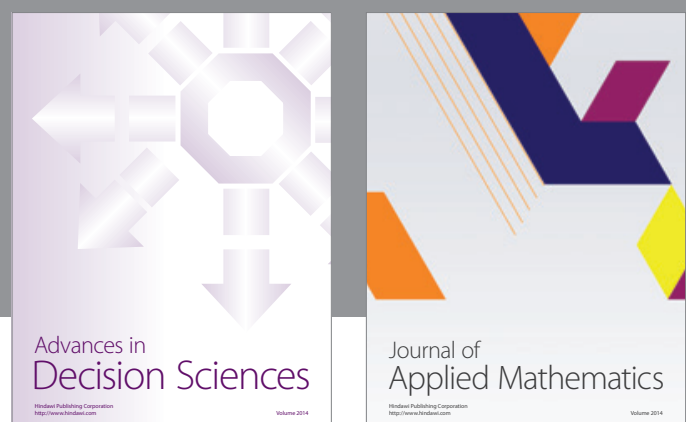

Journal of

Applied Mathematics
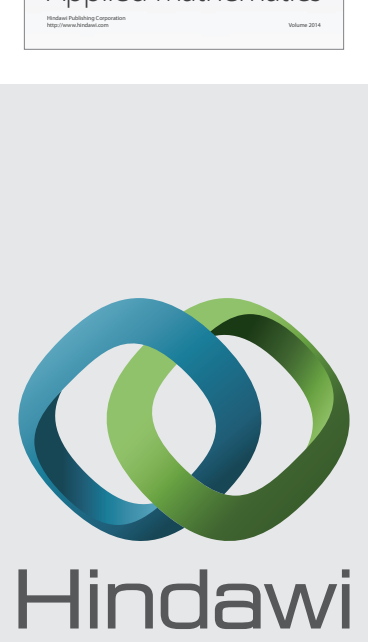

Submit your manuscripts at http://www.hindawi.com
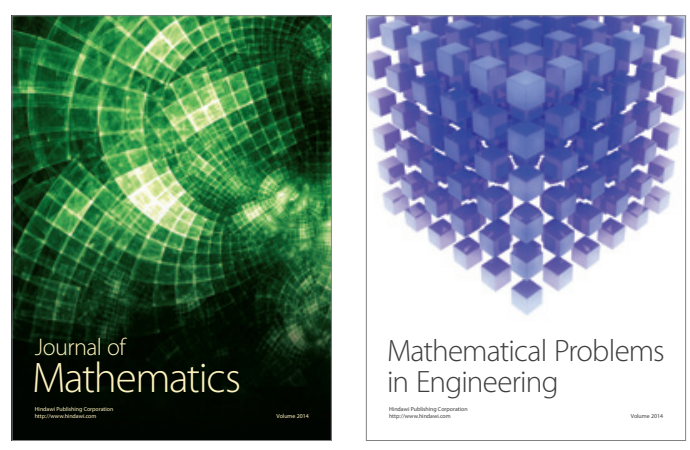

Mathematical Problems in Engineering
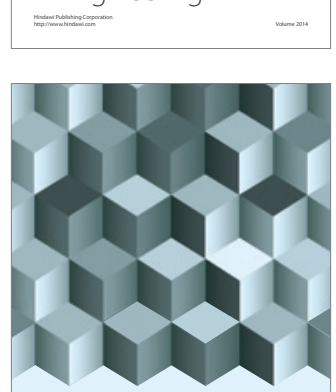

Journal of

Function Spaces
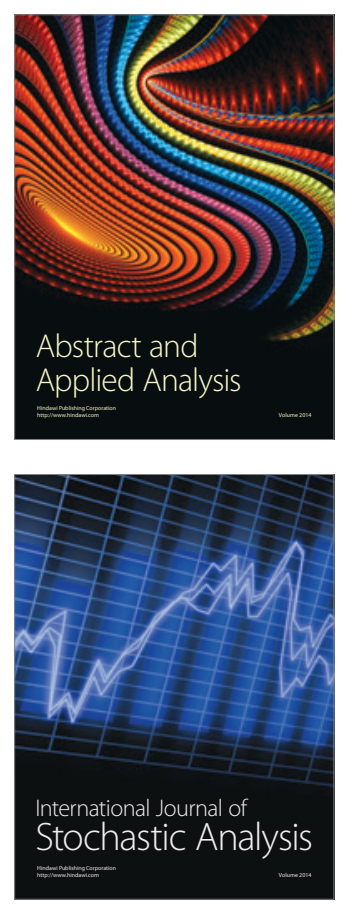

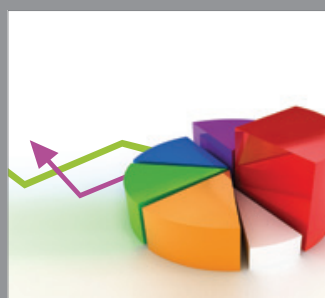

ournal of

Probability and Statistics

Promensencen
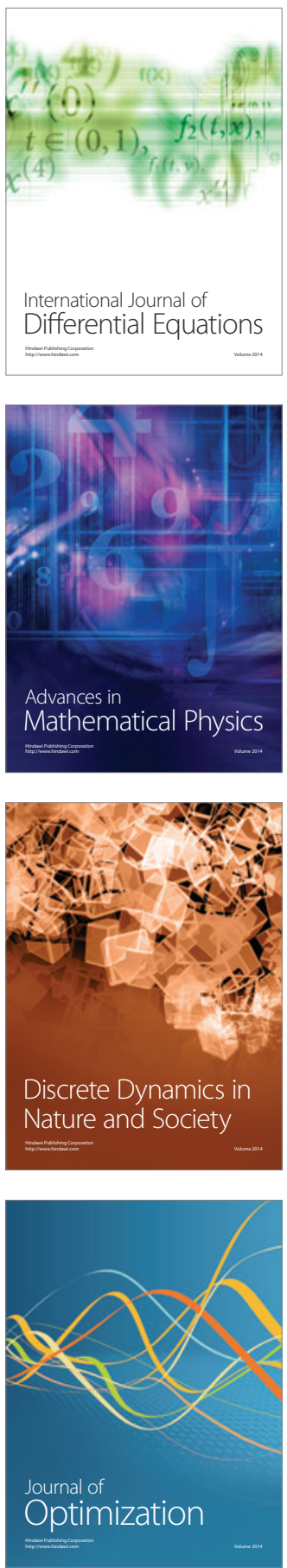